\title{
The contribution of sidewall light extraction to efficiencies of polygonal light-emitting diodes shaped with laser micromachining
}

\author{
X. H. Wang, P. T. Lai, and H. W. Choi \\ Department of Electrical and Electronic Engineering, The University of Hong Kong, Hong Kong
}

(Received 9 March 2010; accepted 25 May 2010; published online 29 July 2010)

\begin{abstract}
The light extraction efficiencies of InGaN/GaN blue light-emitting diodes (LEDs) of different geometries ranging from a triangle to a decagon have been simulated by ray-tracing. The conventional rectangular LED was found to be the most inefficient among the investigated polygons, and light extraction through the device sidewalls was the key factor. The results were experimentally verified by fabricating LEDs shaped into polygons by nanosecond-pulsed laser micromachining, which proved the simulated results. The mechanism of light extraction in polygonal LEDs is discussed in detail. () 2010 American Institute of Physics. [doi:10.1063/1.3456445]
\end{abstract}

\section{INTRODUCTION}

The fabrication, processing and dicing of conventional rectangular light-emitting-diode (LED) chips have become routine procedures in laboratories and industry alike. These dies are then traditionally separated by wafer sawing. The noncontact technique of laser micromachining is gradually replacing the mechanical process, tapping on its unrivalled flexibility and improved precision, allowing features of arbitrary shapes to be formed, with finer feature dimensions and tighter tolerances. ${ }^{1-7}$ Low-damage laser ablation, combining the use of short laser pulses together with precision programmable $x-y$ translation, makes possible the generation of novel LED geometries including polygons, circular disks and even freeform structures. In this work, we explore and examine geometrical possibilities other than conventional rectangular structures for LEDs. Simulation results of light extraction from polygonal structures ranging from a triangle to a decagon are discussed; actual LED chips of these geometries shaped by laser micromachining are demonstrated. Being a macroscale process, chip-shaping can be adopted in conjunction with other strategies employing microstructures and/or nanostructures for enhancing light extraction such as surface texturing ${ }^{8,9}$ photonic crystals, ${ }^{10-12}$ microlens arrays, ${ }^{13-15}$ and pyramidal reflectors ${ }^{16}$ among others.

Laser micromachining with various types of laser sources, including excimer and diode-pumped solid-state lasers, have typically been employed for wafer dicing and device separation in a back-side scribing mode whereby the laser radiation is incident onto the sapphire substrate. ${ }^{4,17}$ The mechanical hardness and optical transparency of sapphire makes it difficult for complete machining through the entire wafer thickness in a single pass, and postmachining cleaving is often required for thick wafer separation. Another problem with deep laser ablation on sapphire substrate is the increasing risk of radiation damage on the epitaxial-grown $\mathrm{GaN}$ layers due to forward light scattering from the sidewall of the kerf. It has been reported that back-side scribing tends to induce more leakage in the devices especially for longer laser wavelength. ${ }^{18}$ In our laser micromachining setup, a 349 $\mathrm{nm}$ pulsed UV laser is used for die separation from $\mathrm{GaN}$
LED wafers. A front-side machining scheme is employed to avoid damage to the active-layer as well as to achieve higher precision with beam alignment.

\section{SIMULATION RESULTS AND DISCUSSION}

Ray-tracing simulations using the software TRACEPRO is performed on ideal $n$-sided polygonal LED models from triangle $(n=3)$ to decagon $(n=10)$. All surfaces of the LED model are modeled to be of optical quality. Light radiates from the multiquantum wells (MQWs) with a uniform pattern and travels through the materials and across interfaces according to Snell's law. The optical parameters of the materials adopted in this simulation are the same as those in our previous report. ${ }^{19}$ All the LED models have vertical sidewalls and have equal top surface area and thus light emission region. The simulated light extraction efficiencies of the polygonal LEDs, defined as the optical flux or power extracted from the LED divided by that emitted from the MQW (set at $50 \mathrm{~mW} / 50000$ rays for this simulation) are summarized in Table I. The most significant observation is that a rectangular LED exhibits lower extraction efficiency by about $10 \%$ to $15 \%$ compared to those of other polygons. This is an interesting observation as the rectangular LED is invariably adopted in most LED products. Differences between sidewall extraction rates are found to be the source of enhancement for the nonconventional polygon LEDs. According to a

TABLE I. Simulated light extraction ratio of polygonal LED models (modeled to be $250 \mu \mathrm{m}$ in thickness, $0.57 \mu \mathrm{m}^{2}$ surface area).

\begin{tabular}{cccc}
\hline \hline Sidewall No. & $\begin{array}{c}\text { Top surface } \\
(\%)\end{array}$ & $\begin{array}{c}\text { Sidewall } \\
(\%)\end{array}$ & $\begin{array}{c}\text { Total } \\
(\%)\end{array}$ \\
\hline 3 & 7.29 & 7.78 & 15.07 \\
4 & 7.29 & 5.68 & 12.98 \\
5 & 7.29 & 7.80 & 15.09 \\
6 & 7.29 & 7.10 & 14.39 \\
7 & 7.29 & 7.25 & 14.54 \\
8 & 7.29 & 7.14 & 14.43 \\
9 & 7.29 & 7.15 & 14.44 \\
10 & 7.29 & 6.82 & 14.11 \\
\hline \hline
\end{tabular}




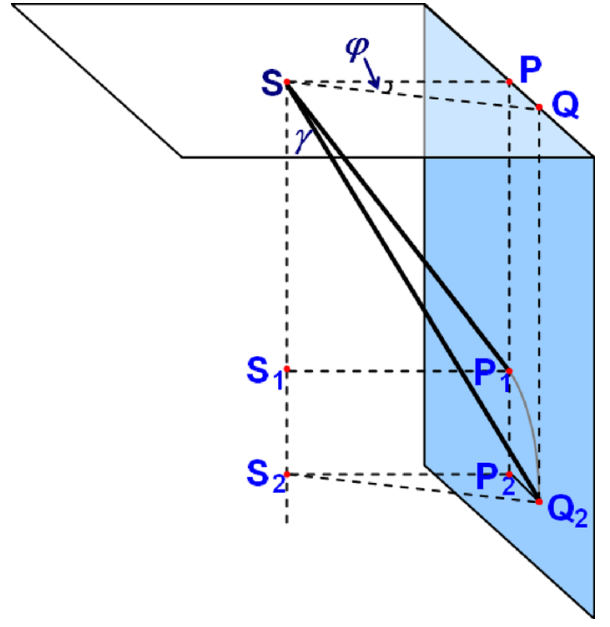

FIG. 1. (Color online) A schematic diagram which illustrates two light rays $\mathrm{SP}_{1}$ and $\mathrm{SQ}_{2}$ emitted from a point source $S$ from the top surface, both making an angle $\gamma$ with respect to the vertical direction. The projection of $\mathrm{SP}_{1}$ onto the top surface $\mathrm{SP}$ is perpendicular to the sidewall while the projection of $\mathrm{SQ}_{2}(\mathrm{SQ})$ makes an angle $\varphi$ to the normal of the sidewall.

simple model based on total internal reflection (TIR), if a light ray reaches the sidewall at an incident angle larger than the critical angle defined by the refractive index difference between sapphire and air, the light ray will be totally reflected and when the same ray encounters another sidewall, the incident angle may change if the two sidewalls are not parallel to each other. In the case of a rectangle, a light ray can make two incident angles with the sidewalls, and the light ray is likely get extracted if either of the two angles falls within the escape cone. In the case of a triangular structure, a light ray can generally make three different incident angles because none of the sidewall facets runs parallel to another. This roughly explains why a triangular LED can provide higher sidewall light extraction efficiency than a rectangular device. The inefficiency of parallel facet pairs is also verified by the simulation results, whereby extraction from LEDs with an odd number of sidewalls is generally higher than those with an even number of sidewalls. However, the question remaining to be answered is why the sidewall extraction of polygons with more sidewall facets does not provide further enhancement even for odd number of sidewall facets.

From a two-dimensional polygonal LED top-view diagram, a point light source irradiates light rays uniformly in all directions across a full $2 \pi$ angle range. The angle $\varphi$ that a light ray makes with the normal of a specific sidewall facet is equal to the incident angle on the sidewall, which is valid in the range of $[-\pi / 2, \pi / 2]$. Due to TIR confinement, only a small fraction of the rays within the range $\left[-\varphi_{c}, \varphi_{c}\right]$ can be transmitted/extracted directly, while rays in the remaining range of angles have to be totally reflected, where $\varphi_{c}$ is the projected critical angle on the plane parallel to the top surface of the device. It is related to the vertical inclination angle $\gamma$, as illustrated in Fig. 1, and the refractive index $n$ of sapphire or $\gamma^{\prime}$ and $n^{\prime}$ in GaN through Eq. (1)

$$
\varphi_{c}=\arccos \left(\frac{\sqrt{1-1 / n^{2}}}{\sin \gamma}\right)=\arccos \left(\frac{\sqrt{n^{2}-1}}{n^{\prime} \sin \gamma^{\prime}}\right) .
$$

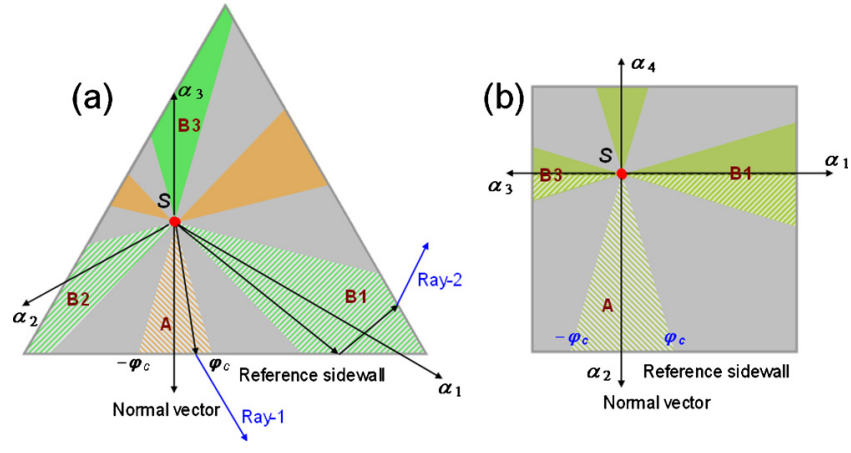

FIG. 2. (Color online) (a) Diagram showing light extraction angular sectors of a triangular LED. Each side (take the reference sidewall, for example) has a direct $\left(\mathrm{A}, 2 \varphi_{c}\right)$ and two shared indirect extraction sectors $(\mathrm{B} 1$ and $\mathrm{B} 2$, $\left.2 \varphi_{c}\right)$; (b) diagram showing light extraction angular sectors of a rectangular LED. Each side has a direct extraction sector $\left(\mathrm{A}, 2 \varphi_{c}\right)$ and two halves of indirect extraction sectors $\left(\mathrm{B} 1\right.$ and $\left.\mathrm{B} 3,2 \varphi_{c}\right)$.

In the case of an n-sided polygon, the angle between any sidewall and the reference sidewall is related to $n$ by Eq. (2), where $\mathrm{k}=1,2, \ldots \mathrm{n}$, such that the two neighboring sidewalls with respect to the reference sidewall corresponds to $\mathrm{k}=1$ and n-1,

$$
\alpha_{k}=\left(1-\frac{2 k}{n}\right) \pi
$$

The incident angle of the reflected light onto the $k$-th sidewall is $\alpha_{k}-\varphi$. Therefore, if $\varphi$ falls within the interval $\left[\alpha_{k}\right.$ $\left.-\varphi_{c}, \alpha_{k}+\varphi_{c}\right]$, the corresponding reflected light can be extracted from the $k$-th sidewall because the second incident angle would still be within the range of $\left[-\varphi_{c}, \varphi_{c}\right]$.

Figure 2(a) shows the angular light extraction pattern for a triangle from a specified point source at site $S$; the vectors $\alpha_{k}$ are marked on the diagram with reference to the surface normal vector of the reference sidewall. Taking the reference sidewall as an example, among the effective angular region $[-\pi / 2, \pi / 2]$, there are three sectors that contribute to light extraction, each of which spans across an angular range of $2 \varphi_{c}$. Section A, shaded in yellow, corresponds to the direct extraction region, while the two other sections B1 and B2 shaded in green contribute to light extraction from neighboring side facets after a single reflection from the reference sidewall or vise versa. Ray-1 and ray-2 illustrates two light paths corresponding to direct and indirect extraction scenarios, respectively. Light rays beyond the allowed sectors as shaded in gray cannot be extracted and are eventually absorbed. This analysis can be applied to the other two sidewalls with identical results. Consequently, each sidewall possesses a direct extraction sector together with two indirect extraction sectors that are shared by the neighboring sidewalls. Considering the overlap of the indirect extraction sectors, the total angular span for light extraction is $12 \varphi_{c}$, which is proportional to the total sidewall light extraction ratio.

Applying a similar analysis to the rectangular geometry, the result is depicted in Fig. 2(b), whereby the slash-shaded halves of regions $\mathrm{B} 1$ and $\mathrm{B} 3$ represent indirect extraction after reflection by the reference sidewall. A total angular span of $8 \varphi_{c}$ is responsible for the sidewall light extractions. Also, the direct extraction sector is no longer exclusive as in a 
TABLE II. Evaluation of direct, indirect, and total light extractions from triangular and rectangular LEDs. It is assumed that direct extraction coefficient $E^{D}$ is 1 , and indirect extraction coefficient $E^{I}$ is estimated to be 0.83 due to reabsorption in $\mathrm{InGaN}$.

\begin{tabular}{lcc}
\hline \hline Extraction type & Triangle & Rectangle \\
\hline Direct extraction & $\frac{6 \varphi_{c}}{2 \pi} \cdot \Delta \cdot E^{D}$ & $\frac{8 \varphi_{c}}{2 \pi}\left[1+\frac{\log \left(\cos \varphi_{c}\right)}{2 \varphi_{c}}\right] \cdot \Delta \cdot E^{D}$ \\
Indirect extraction & $\frac{6 \varphi_{c}}{2 \pi} \cdot \Delta \cdot E^{I}$ & $\frac{8 \varphi_{c}}{2 \pi}\left[-\frac{\log \left(\cos \varphi_{c}\right)}{2 \varphi_{c}}\right] \cdot \Delta \cdot E^{I}$ \\
Total sidewall extraction & $\frac{6 \varphi_{c}}{2 \pi} \cdot \Delta\left(E^{D}+E^{I}\right)$ & $\sim \frac{8 \varphi_{c}}{2 \pi} \cdot \Delta \cdot E^{D}$ \\
\hline
\end{tabular}

triangle, but overlaps with the indirect sectors of the neighboring sidewall. Due to this factor, the triangular geometry is capable of extracting about $50 \%$ more rays from its sidewalls compared to a rectangle assuming that indirect and direct extractions are equally efficient. Similar observations have been reported by others. ${ }^{20}$ However, the simulated enhancement factor lies in the range of $35 \%-40 \%$ depending on the fill ratio of the active region due to the fact that direct extractions are more efficient as reflections are incurred in indirect extraction, leading to a prolonged travel path and thus a higher probability of reabsorption in $\mathrm{InGaN}$.

For $n>4$ polygons, the geometries have more angular extraction sectors and thus the probability of overlap between the angular sectors increases, whether direct or indirect. With increasing number of sidewall facets, the observation angle of a facet from an emission point becomes smaller and smaller, especially toward the ends of that facet. That implies that a significant portion of light rays emitted from the point source cannot reach the target facet directly but is blocked midway by other facets. Although the total number of angular sectors is increased, the effects of multiple reflections dominate, thereby light reabsorption by InGaN rises significantly counteracting the benefits of increased angular extraction sectors. One should also take into account the effective area of an extraction mode (direct or indirect) as well as the span of the extraction angle. As an example, Table II lists the amounts of extraction from a triangular LED and a rectangular LED where the effective area is given based on the calculations with reference to Fig. 3.

The total extraction region defined by the isosceles triangle APB of Fig. 3(a) overlaps with the entire area of the triangular LED ABC and a portion of its sidewall mirrored images $\mathrm{A}^{\prime} \mathrm{BC}$ (shaded in yellow); for direct extraction, light rays are extracted through side $\mathrm{AB}$ without intermediate reflections by neighboring sidewalls $\mathrm{BC}$ or $\mathrm{AC}$, as illustrated by the rays from a point light source $S$ propagating in the region bordered by $\mathrm{SE}$ and $\mathrm{SF}$. For indirect extraction, a reflection by another sidewall is involved, thus the light ray would appear to emit from its mirrored image. It can be seen in Fig. 3(a) that all direct extraction rays and a portion of the indirect extraction rays (reflected by neighboring sidewall) are completely extracted as they are emitted from within the total extraction region, such as rays emitted from points $S$ (direct) and $S^{\prime}$ (indirect), where $S^{\prime}$ is the imaged point of $S$ mirrored by the sidewall BC. The final extraction is esti-

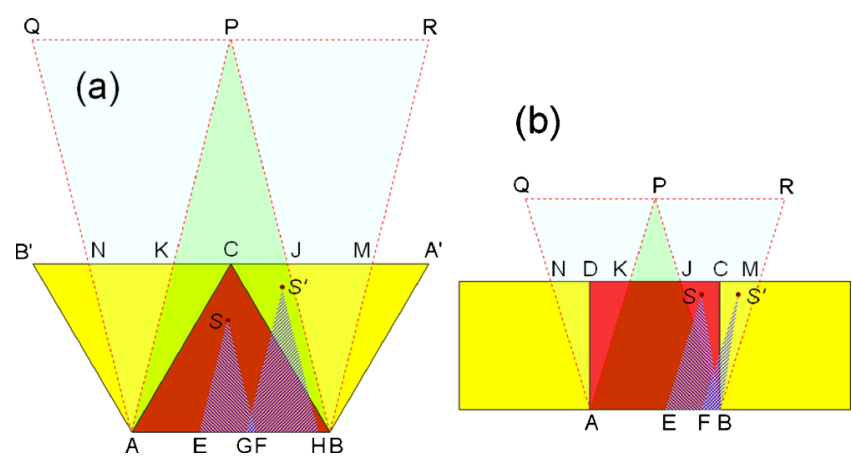

FIG. 3. (Color online) (a) The triangular LED ABC together with part of the indirect extraction region (A'BC and $\mathrm{B}^{\prime} \mathrm{AC}$ ) is fully covered by the total extraction zone APB. (b) In contrast, a rectangular LED is not completely covered by the total extraction zone APB and only a minor portion of indirect light extraction region is covered by the partial extraction zone within PAQ and PBR. Triangles APB, PAQ, and PBR are isosceles triangles with a vertex angle of $2 \varphi_{c}$.

mated by the product of three factors: first, the span of extraction angle as illustrated in Fig. 2, second the effective emission area which is weighted by the extent of overlap between the extraction angular sector of a sidewall facet and the observation angle of the said facet from an emission point, and finally the extraction coefficient $E^{D}$ (direct extraction) or $E^{I}$ (indirect extraction). As the triangular LED surface $\mathrm{ABC}$ overlaps completely with the total extraction region APB, the effective emission area for direct extraction from a triangular LED is that of the entire surface of triangle $\mathrm{ABC}$, which we define to be $\Delta$ in size. The effective indirect extraction area is calculated by evaluating the overlap between the imaged areas (shaded in yellow) and the extraction regions, which is composed of both the complete extraction portions $\mathrm{BCJ}$ and $\mathrm{ACK}$, and the partial extraction portions $\mathrm{BJM}$ and $\mathrm{AKN}$ that are covered by the partial extraction regions PBR and PAQ, respectively, in Fig. 3(a). The calculated partial extraction rate for BJM and AKN is 0.5 on average by integration across the triangles. Therefore, the effective indirect extraction area sums up to be $\Delta$, equal to that of the direct extraction area. Consequently, the amount of indirect extraction from a triangular LED is comparable to that of direct extraction, neglecting the factor of extraction coefficient as indicated in Table II.

In the case of a rectangular LED, the overlap region between total extraction region $\mathrm{PAB}$ and the rectangle $\mathrm{ABCD}$ is represented by the trapezoidal portion ABJK in Fig. 3(b); light emitted from beyond the trapezoid region is only partially extracted, such as the point sources $S$ and $S^{\prime}$. The amount of direct and indirect extractions from a rectangular LED is calculated using the same method and listed in Table II for comparison. According to Eq. (1), the maximum $\varphi_{c}$ is $34.2^{\circ}$ and the corresponding maximum indirect extraction in a rectangular LED accounts for about $7 \%$ of the total extraction. Therefore, the effective indirect extraction would be less than $7 \%$ averaging on the range of valid $\gamma^{\prime}$. As a result, the indirect extractions in a rectangular LED constitute a much smaller fraction than the direct extractions; while in the case of a triangular LED indirect extraction constitute almost half of the total extractions. For $n>4$ polygon LED, although the sum of extraction angle fraction is expected to 


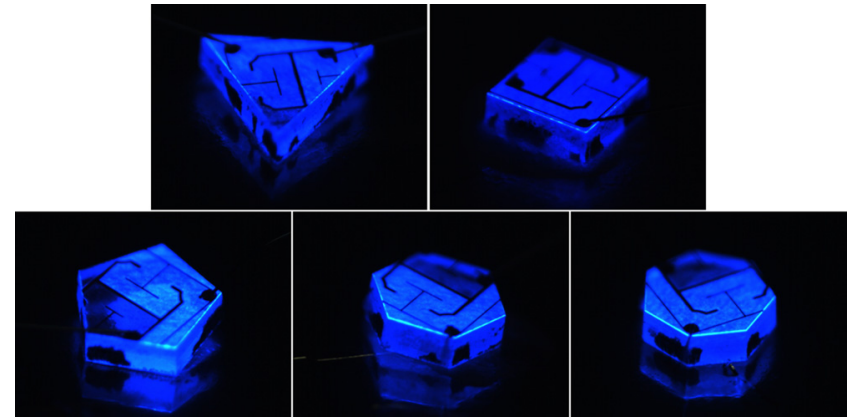

FIG. 4. (Color online) Optical microscopy images of polygonal LED chips operated at a bias voltage of $2.5 \mathrm{~V}$; the center wavelength of emission is 470 nm.

be $2 \mathrm{n} \varphi_{c} / 2 \pi$, the effective area reduces significantly with an increase in sidewall number, as a result the extraction ratio does not increase linearly with $n$. For odd numbers of side facets, the maximum sidewall extraction ratio is achieved to be $7.80 \%$ with a pentagon, and for even numbers, the maximum of $7.14 \%$ is obtained with an octagon. Although the hexagon is not the most efficient geometry among the polygons, the fact that hexagons can be closed-packed makes it most suitable for commercial applications, as the loss of chip space is minimized allowing for maximum device density on a wafer.

\section{DEVICE FABRICATION AND CHARACTERIZATION}

The results are further verified experimentally by cutting polygonal LEDs with different number of sides by laser micromachining, using a $349 \mathrm{~nm}$ nanosecond pulsed UV laser as the beam source. Standard large area $\left(1 \times 1 \mathrm{~mm}^{2}\right)$ LED chips were fabricated on an $\mathrm{InGaN} / \mathrm{GaN}$ blue LED wafer with emission wavelength of $470 \mathrm{~nm}$ using standard microfabrication techniques. Polygonal chips of equal emission areas were cut from within these squarish chips. To facilitate alignment of the beam and to minimize the damaged regions on the semiconductor layers, we adopted front-side machining with the front surface of the GaN-based LED wafer protected with a photoresist protective and sacrificial layer. The process conditions have been optimized with the laser beam accurately focused on the top surface of the wafer to attain a small spot size of $\sim 10 \mu \mathrm{m}$. For a fair comparison of light extraction efficiencies between different polygonal geometries, the chips have equal emission areas of $0.57 \mathrm{~mm}^{2}$. The photoresist layer is then striped by acetone after laser micromachining and the samples cleaned with a diluted $\mathrm{HCl}$ solution. The chips are die-bonded onto transistor outline headers followed by Al wire-bonding. Figure 4 shows packaged polygonal LEDs from triangle $(n=3)$ to heptagon $(n=7)$ operated at a low voltage of $2.5 \mathrm{~V}$.

Electrical measurements of the packaged LED devices indicate unchanged I-V characteristics compared with the asfabricated devices. No obvious leakage ramp is observed for all the devices at below $2.5 \mathrm{~V}$ whether measured before or after chip separation. The absence of laser damage can be attributed to well-control of the heat affected zone (HAZ) through a few strategies. First, front side micromachining with the GaN layer facing the laser beam avoids scattering

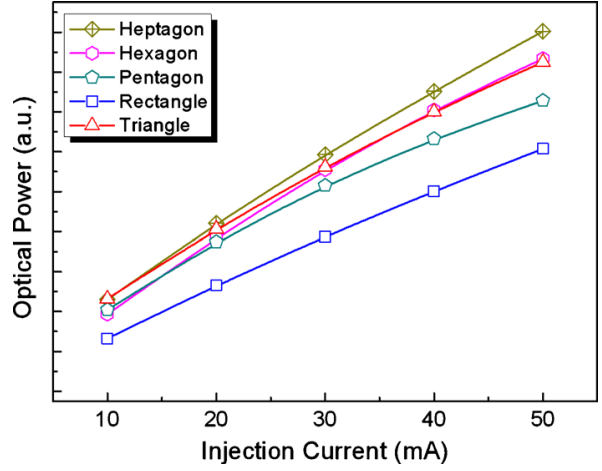

FIG. 5. (Color online) Measured light output power vs injection current (L-I) for laser micromachined polygonal LEDs.

within the sapphire substrate layer, which is highly transparent at $349 \mathrm{~nm}$ wavelength and with much higher ablation threshold than GaN; second the laser beam is tightly focused on the surface of semiconductors and the aspect ratio of the kerf is found to be maximized under such optimization, minimizing lateral machining.

The optical powers of the packaged devices were measured by placing the devices within a 2 in. integrating sphere, and the light from the output port channeled to a calibrated spectrometer via an optical fiber. The results are shown in the light intensity versus current (L-I) characteristics plot in Fig. 5 , which clearly shows that the rectangular LED emits with the lowest intensity at all currents. To ensure reliability and reproducibility of the data, the L-I data was collected from more than one device for each geometry. The statistical variations in measured light output at $20 \mathrm{~mA}$ injection current, together with the simulation results, are plotted in Fig. 6. Once again, the advantages of polygonal geometries are evident.

\section{CONCLUSION}

Conventional rectangle LEDs have been proved to be incompetent for light extraction compared to other polygonal geometries. With the triangular geometry, indirect light extraction via TIR on neighboring sidewalls contributes to enhancement of light extraction over a rectangle. For polygons with larger sidewall numbers $n>4$, the reduction in effective

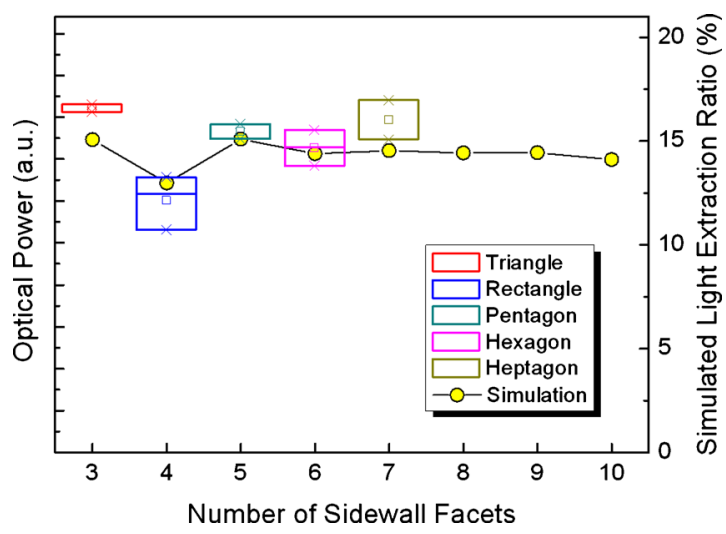

FIG. 6. (Color online) Measured light output from a range of polygonal LEDs biased at $20 \mathrm{~mA}$ ( $\mathrm{n}=3$ to 7$)$, together with their simulated light extraction ratios. 
light extraction area counteract the increase in allowed angular sectors for both direct and indirect extraction. Enhanced light extraction from nonconventional polygonal LEDs is verified experimentally with devices shaped by laser micromachining. Taking into account the device packing density, LEDs of hexagon geometry is proposed as a promising alternative choice that makes best use of the wafer space while optimizing efficiencies of LED devices.

\section{ACKNOWLEDGMENTS}

This work was supported by a GRF grant of the Research Grant Council of Hong Kong (Project No. HKU 7118/ 09E).

${ }^{1}$ V. V. Kancharla, K. K. Hendricks, and S. C. Chen, Proc. SPIE 4557, 220 (2001).

${ }^{2}$ F. Yoshino, H. B. Zhang, and A. Arai, J. Laser Micro/Nanoeng. 4, 212 (2009).

${ }^{3}$ A. Y. Arai, G. C. Cho, J. Z. Xu, F. Yoshino, H. B. Zhang, J. Bovatsek, and M. Yoshida, U.S. Patent Application No. 20100025387.

${ }^{4}$ T. P. Glenn, S. Webster, and R. D. Hollaway, U.S. Patent No. 6399463.

${ }^{5}$ A. A. Tseng, Y. T. Chen, and K. J. Ma, Opt. Lasers Eng. 41, 827 (2004).

${ }^{6}$ G. P. Vakanas, A. A. Tseng, and P. Winer, J. Laser Appl. 14, 185 (2002).

${ }^{7}$ H. Y. Zheng, E. Gan, and G. C. Lim, Opt. Lasers Eng. 36, 355 (2001).
${ }^{8}$ S. X. Jin, J. Li, J. Y. Lin, and H. X. Jiang, Appl. Phys. Lett. 77, 3236 (2000).

${ }^{9}$ H. W. Choi, C. W. Jeon, and M. D. Dawson, IEEE Photon. Technol. Lett. 16, 33 (2004).

${ }^{10}$ T. Kim, A. J. Danner, and K. D. Choquette, Electron. Lett. 41, 1138 (2005).

${ }^{11}$ J. J. Wierer, M. R. Krames, J. E. Epler, N. F. Gardner, J. R. Wendt, M. M. Sigalas, S. R. J. Brueck, D. Li, and M. Shagam, Proc. SPIE 5739, 102 (2005)

${ }^{12}$ K. McGroddy, A. David, E. Matioli, M. Iza, S. Nakamura, S. DenBaars, J. S. Speck, C. Weisbuch, and E. L. Hu, Appl. Phys. Lett. 93, 103502 (2008).

${ }^{13}$ Y. K. Ee, R. A. Arif, N. Tansu, P. Kumnorkaew, and J. F. Gilchrist, Appl. Phys. Lett. 91, 221107 (2007).

${ }^{14}$ H. W. Choi, C. Liu, E. Gu, G. McConnell, J. M. Girkin, I. M. Watson, and M. D. Dawson, Appl. Phys. Lett. 84, 2253 (2004).

${ }^{15}$ Y. K. Ee, P. Kumnorkaew, R. A. Arif, H. Tong, J. F. Gilchrist, and N. Tansu, Opt. Express 17, 13747 (2009).

${ }^{16}$ J. Q. Xi, H. Luo, A. J. Pasquale, J. K. Kim, and E. F. Schubert, IEEE Photon. Technol. Lett. 18, 2347 (2006).

${ }^{17}$ D. Ulieru, A. Tantau, E. Ulieru, I. Cernica, A. Matei, V. Schiopu, and F. Pistritu, CAS International Semiconductor Conference Proceedings, 1-2, 155 (2007).

${ }^{18}$ E. K. Illy, M. Knowles, E. Gu, and M. D. Dawson, Appl. Surf. Sci. 249, 354 (2005)

${ }^{19}$ X. H. Wang, W. Y. Fu, P. T. Lai, and H. W. Choi, Opt. Express 17, 22311 (2009).

${ }^{20}$ J. Y. Kim, M. K. Kwon, J. P. Kim, and S. J. Park, IEEE Photon. Technol. Lett. 19, 1865 (2007). 